\begin{tabular}{|c|l|}
\hline Title & Shape of C-13 studied by the real-time evolution method \\
\hline Author(s) & Shin, S.; Zhou, B.; Kimura, M. \\
\hline Citation & $\begin{array}{l}\text { Physical Review C, 103(5), 054313 } \\
\text { https://doi.org/10.1103/PhysRevC.103.054313 }\end{array}$ \\
\hline Issue Date & 2021-05-19 \\
\hline Doc URL & http://hdl.handle.net/2115/82215 \\
\hline Rights & @2021 A merican Physical Society \\
\hline Type & article \\
\hline File Information & PhysRevC.103-5_054313.pdf \\
\hline
\end{tabular}

Instructions for use 


\title{
Shape of ${ }^{13} \mathrm{C}$ studied by the real-time evolution method
}

\author{
S. Shin $\odot,{ }^{1}$ B. Zhou $\odot,{ }^{2}$ and M. Kimura ${ }^{1,3,4, *}$ \\ ${ }^{1}$ Department of Physics, Hokkaido University, Sapporo 060-0810, Japan \\ ${ }^{2}$ Institute of Modern Physics, Fudan University, Shanghai 200433, China \\ ${ }^{3}$ Nuclear Reaction Data Centre, Hokkaido University, Sapporo 060-0810, Japan \\ ${ }^{4}$ Research Center for Nuclear Physics (RCNP), Osaka University, Ibaraki 567-0047, Japan
}

(Received 27 December 2020; accepted 7 May 2021; published 19 May 2021)

\begin{abstract}
Background: Recently, Bijker et al. [Phys. Rev. Lett. 122, 162501 (2019)] explained the rotation-vibration spectrum of ${ }^{13} \mathrm{C}$ by assuming a triangular nuclear shape with $D_{3 h}^{\prime}$ symmetry.

Purpose: The purpose of this work is to test the shape and symmetry of ${ }^{13} \mathrm{C}$ based on a microscopic nuclear model without assumption of nuclear shape.

Method: We have applied the real-time evolution method to ${ }^{13} \mathrm{C}$. By using the equation-of-motion of clusters, the model describes the $3 \alpha+n$ system without any assumption of symmetry.

Results: REM described the low-lying states more accurately than the previous cluster model studies. The analysis of the wave functions showed that the ground band has approximate triangular symmetry, while the excited bands deviate from it.

Conclusion: This work confirmed that the ground band has the intrinsic structure with the triangular arrangement of three $\alpha$ particles.
\end{abstract}

DOI: 10.1103/PhysRevC.103.054313

\section{INTRODUCTION}

Carbon isotopes have been an important subject in nuclear cluster physics as they manifest a rich variety of cluster phenomena. The Hoyle state (the $\mathrm{O}_{2}{ }^{+}$state of ${ }^{12} \mathrm{C}$ ) exhibits one of the most interesting clustering aspects Bose-Einstein condensate (BEC) of three $\alpha$ particles [1]. The structure of the Hoyle state and its analogous states in neighboring nuclei has been one of the major topics in recent decades [2-14]. In the highly excited region of carbon isotopes, a different type of clustering, the linear chain of $\alpha$ particles, has also been intensively discussed [15-28]. Among the carbon isotopes, ${ }^{13} \mathrm{C}$ is of particular importance as the system composed of three $\alpha$ particles (bosons) plus a valence nucleon (fermion). The Hoyle analog state in ${ }^{13} \mathrm{C}$, the BEC of three $\alpha$ particles with a neutron as an impurity, and the possible formation of the linear-chain structure assisted by a valence neutron have been main interests for this nucleus [29-37].

Recently, apart from these studies, Bijker et al. [38] proposed a different interpretation for the structure of ${ }^{13} \mathrm{C}$ based on the symmetry arguments. They applied the algebraic cluster model (ACM) [39-41] to ${ }^{13} \mathrm{C}$, and assumed triangular $D_{3 h}^{\prime}$ symmetry of the $3 \alpha$ clusters accompanied by a valence neutron. The intrinsic states were classified into three representations of the symmetry group, and each of them forms the rotational band and exhibits unique spectrum and transition strengths. Based on a comparison with available experimental data, they argued that many of the observed ground and excited states can be assigned to these bands, and

*masaaki@nucl.sci.hokudai.ac.jp hence, ${ }^{13} \mathrm{C}$ has triangular $D_{3 h}^{\prime}$ symmetry. This suggests an interesting insight into the structure of carbon isotopes and contradicts the BEC interpretation of the Hoyle state and its analog states. However, the model is based on pure symmetry concepts and the deviation from the triangular symmetry, which must take place in reality, is neglected. Therefore, the symmetry behind the spectrum of ${ }^{13} \mathrm{C}$ and deviation from it should be tested by microscopic models without any assumption of the nuclear shape.

The real-time evolution method (REM) recently proposed by Imai et al. [42] is one of the microscopic cluster models which can examine the shape of nuclei without any assumption of the symmetry. It generates basis wave functions with various cluster configurations by using the equation of motion (EOM) of the Gaussian wave packets. A benchmark calculation showed that REM precisely describes the $3 \alpha$ system including the Hoyle state. Therefore, a natural idea is to extend the method to the non- $N \alpha$ systems [43]. It is noted that REM superposes a massive number of the basis wave functions to describe the cluster systems, and it does not introduce any assumption about the symmetry of nuclear shape and cluster configurations. Therefore, REM is a suitable nuclear model to test if there exists any symmetry in the spectrum of ${ }^{13} \mathrm{C}$. Thus, the aim of this work is twofold. The first is the extension and benchmark of REM for non- $N \alpha$ system, and the second is the verification of the triangular $D_{3 h}^{\prime}$ symmetry in the spectrum of ${ }^{13} \mathrm{C}$.

We organize this paper as follows. In the next section, the framework of REM for the $3 \alpha+n$ system is briefly explained. In Sec. III, the numerical results are presented. Compared to a previous study which used the same Hamiltonian, REM yields deeper binding energies for all bound states and describes the 
$3 \alpha+n$ system more accurately. Based on the $B(E 2)$ strengths, we propose an assignment of the rotational bands and discuss the internal structure of the band member states to examine the triangular $D_{3 h}^{\prime}$ symmetry. It is shown that the ground band member states have the same intrinsic structure, which has triangular arrangement of three $\alpha$ particles. However, it is found that many excited states deviate from a rigid body and fluctuate around the triangular shape. Finally, in the last section, we summarize this work.

\section{THEORETICAL FRAMEWORK}

In this section, we explain the Hamiltonian and framework of the real-time evolution method for the $3 \alpha+n$ system. The Hamiltonian used in this study is given as

$$
\hat{H}=\sum_{i=1}^{13} \hat{t}_{i}+\sum_{i<j}^{13} \hat{v}_{N}\left(r_{i j}\right)+\sum_{i<j}^{13} \hat{v}_{C}\left(r_{i j}\right)-\hat{t}_{\mathrm{c} . \mathrm{m} .},
$$

where $\hat{t}_{i}$ is the kinetic energy of the $i$ th nucleon and $\hat{t}_{\mathrm{c} . \mathrm{m}}$. is the center-of-mass kinetic energy. $\hat{v}_{N}$ and $\hat{v}_{C}$ denote the effective nucleon-nucleon and Coulomb interactions, respectively. For the central part of the nucleon-nucleon interaction, we used Volkov no. 2 force [44] with the exchange parameters, $W=$ $0.4, B=H=0.125$, and $M=0.6$. The G3RS force [45] is used for the spin-orbit part with two choice of the strength, $u_{l s}=1000$ and $2000 \mathrm{MeV}$. The latter value $u_{l s}=2000 \mathrm{MeV}$ was also used by Furutachi et al. [31], and we also adopt the same strength for the sake of comparison. However, as shown later, it does not reproduce the correct ordering of the ground band member states, and hence, we also applied a weaker strength $u_{l s}=1000 \mathrm{MeV}$ for better description of the ground band.

As the basis wave function to describe the $3 \alpha+n$ system, we employ the Brink-Bloch wave function [46], which consists of three $\alpha$ clusters with $(0 s)^{4}$ configuration coupled with a valence neutron,

$$
\begin{aligned}
\Phi\left(\boldsymbol{Z}_{1}, \ldots, \boldsymbol{Z}_{4}\right) & =\mathcal{A}\left\{\Phi_{\alpha}\left(\boldsymbol{Z}_{1}\right) \Phi_{\alpha}\left(\boldsymbol{Z}_{2}\right) \Phi_{\alpha}\left(\boldsymbol{Z}_{3}\right) \Phi_{n}\left(\boldsymbol{Z}_{4}\right)\right\} \\
\Phi_{\alpha}(\boldsymbol{Z}) & =\mathcal{A}\left\{\phi\left(\boldsymbol{r}_{1}, \boldsymbol{Z}\right) \chi_{p \uparrow} \cdots \phi\left(\boldsymbol{r}_{4}, \boldsymbol{Z}\right) \chi_{n \downarrow}\right\} \\
\Phi_{n}(\boldsymbol{Z}) & =\phi(\boldsymbol{r}, \boldsymbol{Z}) \chi_{n \uparrow}, \\
\phi(\boldsymbol{r}, \boldsymbol{Z}) & =\left(\frac{2 v}{\pi}\right)^{3 / 4} \exp \left\{-v(\boldsymbol{r}-\boldsymbol{Z})^{2}\right\}
\end{aligned}
$$

where $\Phi_{\alpha}(\boldsymbol{Z})$ and $\Phi_{n}(\boldsymbol{Z})$ denote the wave packets describing the $\alpha$ cluster and the valence neutron located at $\boldsymbol{Z}$, respectively. In this study, we fix the valence neutron spin to up in the intrinsic frame without loss of generality. The set of three-dimensional vectors $\boldsymbol{Z}_{1}, \ldots, \boldsymbol{Z}_{4}$ is complex numbered and describes positions and momenta of the $3 \alpha+n$ clusters in the phase space. The size parameter $v=1 / 2 b^{2}$ of the $\alpha$ particle is fixed to $b=1.46 \mathrm{fm}$, which reproduces the observed size of an $\alpha$ particle. The same size parameter is also used to describe the valence neutron.

In the REM framework, we use the equation of motion to generate the basis wave functions with various configurations of clusters. From the time-dependent variational principle,

$$
\delta \int d t \frac{\left\langle\Phi\left(\boldsymbol{Z}_{1}, \ldots, \boldsymbol{Z}_{4}\right)|i \hbar d / d t-\hat{H}| \Phi\left(\boldsymbol{Z}_{1}, \ldots, \boldsymbol{Z}_{4}\right)\right\rangle}{\left\langle\Phi\left(\boldsymbol{Z}_{1}, \ldots, \boldsymbol{Z}_{4}\right) \mid \Phi\left(\boldsymbol{Z}_{1}, \ldots, \boldsymbol{Z}_{4}\right)\right\rangle}=0,
$$

one obtains the equation of motion (EOM) for the $3 \alpha+n$ cluster centroids $\boldsymbol{Z}_{1}, \ldots, \boldsymbol{Z}_{4}$,

$$
\begin{gathered}
i \hbar \sum_{j=1}^{4} \sum_{\sigma=x, y, z} C_{i \rho j \sigma} \frac{d Z_{j \sigma}}{d t}=\frac{\partial \mathcal{H}_{\text {int }}}{\partial Z_{i \rho}^{*}}, \\
\mathcal{H}_{\text {int }} \equiv \frac{\left\langle\Phi\left(\boldsymbol{Z}_{1}, \ldots, \boldsymbol{Z}_{4}\right)|\hat{H}| \Phi\left(\boldsymbol{Z}_{1}, \ldots, \boldsymbol{Z}_{4}\right)\right\rangle}{\left\langle\Phi\left(\boldsymbol{Z}_{1}, \ldots, \boldsymbol{Z}_{4}\right) \mid \Phi\left(\boldsymbol{Z}_{1}, \ldots, \boldsymbol{Z}_{4}\right)\right\rangle}, \\
C_{i \rho j \sigma} \equiv \frac{\partial^{2} \ln \left\langle\Phi\left(\boldsymbol{Z}_{1}, \ldots, \boldsymbol{Z}_{4}\right) \mid \Phi\left(\boldsymbol{Z}_{1}, \ldots, \boldsymbol{Z}_{4}\right)\right\rangle}{\partial Z_{i \rho}^{*} \partial Z_{j \sigma}} .
\end{gathered}
$$

By solving this EOM from an arbitrary initial wave function, a set of the vectors $Z_{1}(t), \ldots, Z_{4}(t)$ is obtained as a function of the real-time $t$, which defines the basis wave function $\Phi\left(\boldsymbol{Z}_{1}(t), \ldots, \boldsymbol{Z}_{4}(t)\right)$ at each time.

When we solve the EOM, we add an external field $V_{d}$ to the Hamiltonian,

$$
\begin{aligned}
V_{d} & =v_{d} \sum_{i} f\left(\left|\operatorname{Re} \boldsymbol{Z}_{i}-\boldsymbol{R}_{\mathrm{c} . \mathrm{m} .}\right|\right) \\
f(x) & =(x-a)^{2} \theta(x-a) \\
\boldsymbol{R}_{\text {c.m. }} & =\frac{4}{13} \sum_{i=1}^{3} \operatorname{Re} \boldsymbol{Z}_{i}+\frac{1}{13} \operatorname{Re} \boldsymbol{Z}_{4},
\end{aligned}
$$

where $\theta(x-a)$ is the step function. This external field reflects constituent particles at distance $a$ to prevent them escaping far away. The external field strength is chosen as $v_{d}=$ 1.5 $\mathrm{MeV} / \mathrm{fm}^{2}$ which is strong enough to confine the particles and not too strong to cause numerical error. The spatial size of the confinement is chosen as $a=10 \mathrm{fm}$, and we confirmed other choices of $a$ ( 8 and $12 \mathrm{fm}$ ) do not change the numerical results presented in the following.

Once we obtain a set of basis wave functions, we perform the generator coordinate method (GCM) calculation by superposing them after the projection of the parity and angular momentum,

$$
\Psi_{M}^{J \pi}=\int_{0}^{T_{\max }} d t \sum_{K=-J}^{J} \hat{P}_{M K}^{J \pi} f_{K}(t) \Phi\left(\boldsymbol{Z}_{1}(t), \ldots, \boldsymbol{Z}_{4}(t)\right),
$$

where $\hat{P}_{M K}^{J \pi}$ is the parity and the angular momentum projection operator,

$$
\hat{P}_{M K}^{J \pi}=\frac{2 J+1}{8 \pi^{2}} \int d \Omega \mathcal{D}_{M K}^{J *}(\Omega) \hat{R}(\Omega) \frac{1+\pi \hat{P}_{x}}{2} .
$$

In the practical calculation, the integral in Eq. (13) is discretized as

$$
\Psi_{M}^{J \pi}=\sum_{i K} \hat{P}_{M K}^{J \pi} f_{i K} \Phi_{i},
$$

where $\Phi_{i}$ is an abbreviation for $\Phi\left(Z_{1}(t), \ldots, Z_{4}(t)\right)$. The amplitude $f_{i K}$ and eigenenergy are determined by solving the Hill-Wheeler equation $[47,48]$. 


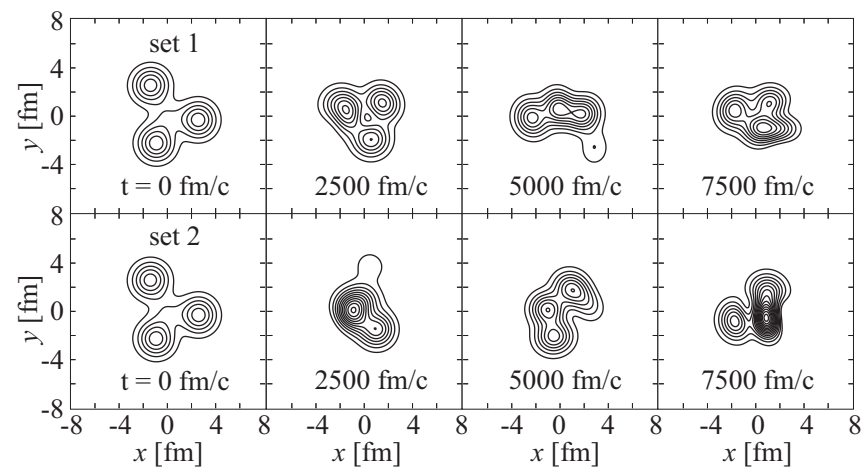

FIG. 1. The snapshots of the intrinsic density distributions obtained by the real-time evolution. The top (bottom) panels show the wave functions from the ensemble set 1 (set 2).

\section{RESULTS AND DISCUSSION}

\section{A. Time evolution of the $3 \alpha+n$ system}

The numerical calculations were performed according to the following procedure. First, using pure imaginary-time $\tau=i t$ in Eq. (7), we calculate the minimum intrinsic energy that is found to be $-83.1 \mathrm{MeV}$. Then, we generate the wave functions with the intrinsic excitation energy $E_{\text {int }}^{*}$ using the same equation. We have tested several excitation energies and used $E_{\text {int }}^{*}=30 \mathrm{MeV}$ in this work as it gives the best convergence of the GCM calculation. Using these wave functions as the initial condition at $t=0$, we calculate the time evolution of the $3 \alpha+n$ system. The total propagation time was set to $10000 \mathrm{fm} / \mathrm{c}$, and the wave functions are recorded at every 33 $\mathrm{fm} / \mathrm{c}$. Consequently, an ensemble of the 300 wave functions is generated. By using different initial wave functions at $t=0$, we generated two ensembles which we call sets 1 and 2 .

Several snapshots of the wave functions from these ensembles are shown in Fig. 1. Note that the wave functions of sets 1 and 2 at $t=0 \mathrm{fm} / \mathrm{c}$ have different momenta of clusters, although they have almost the same spatial distributions. Consequently, the sets 1 and 2 show the different results of the time evolution. We also note that various nuclear shapes with different cluster configurations naturally emerge from the EOM. In some cases, $3 \alpha$ particles are close to each other and the valence neutron is apart from them. In other cases, $2 \alpha$ particles and the valence neutron are close to each other, and an $\alpha$ particle is apart from others describing ${ }^{9} \mathrm{Be}^{*}+\alpha$ like configurations. In this manner, the ensembles of the basis wave functions were prepared without any assumption of the spatial symmetry.

\section{B. The calculated full spectrum}

The generated wave functions are superposed to diagonalize the Hamiltonian. To confirm the convergence of the calculation, Fig. 2 shows the energies and radii of the $1 / 2_{1}{ }^{-}$ and $5 / 21^{+}$states, which are the lowest negative- and positiveparity states, as functions of the propagation time $T_{\max }$. The energy and radius of the ground state $\left(1 / 2_{1}{ }^{-}\right.$state) show fast convergence and both sets reach almost the identical values. Thus, the obtained GCM wave functions are converged well independent of the initial wave functions. The figure also
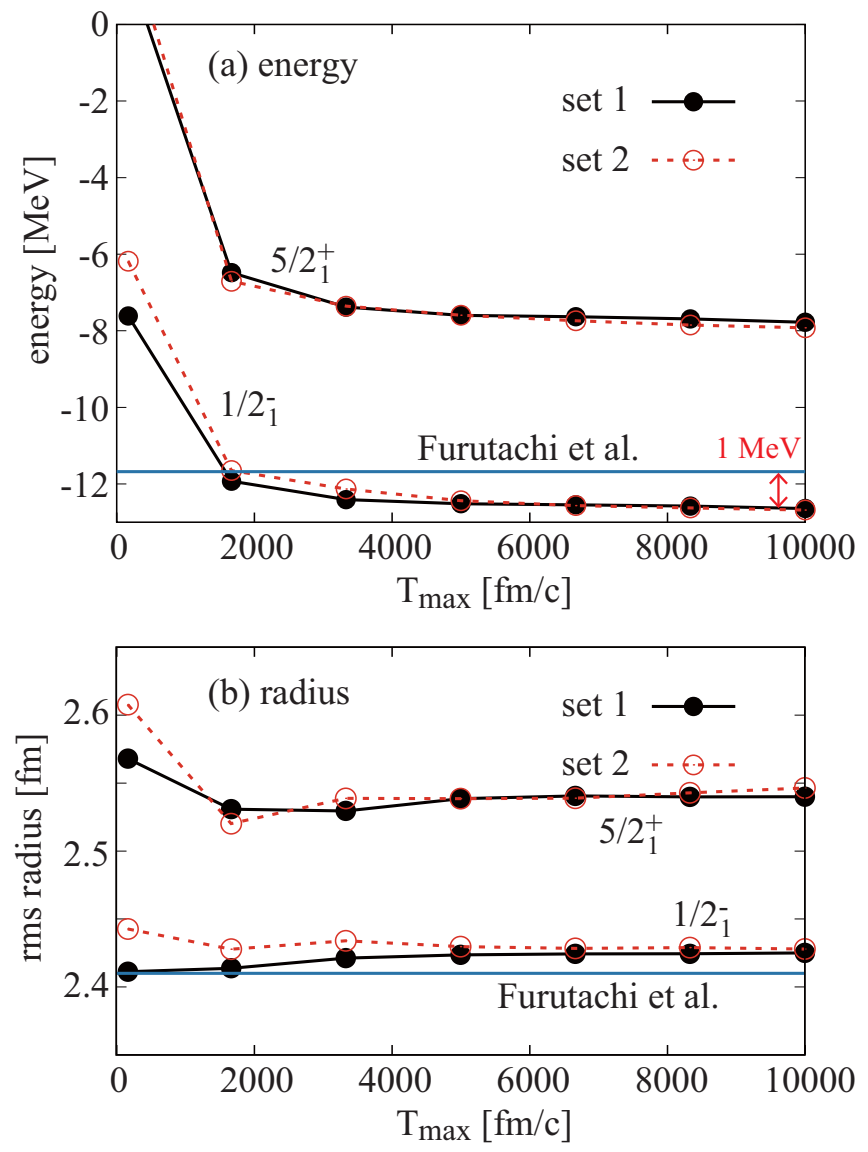

FIG. 2. The energies and radii of the $1 / 2_{1}{ }^{-}$and $5 / 2{ }_{1}{ }^{+}$states obtained from sets 1 and 2 as a function of the total propagation time $T_{\max }$. The strength of the spin-orbit potential $u_{l s}=2000 \mathrm{MeV}$ was adopted. The result for the $1 / 2_{1}{ }^{-}$state obtained in Ref. [31] are denoted by blue lines.

shows that REM yields approximately $1 \mathrm{MeV}$ deeper binding energy of the $1 / 2_{1}{ }^{-}$state than the previous study by Furutachi et al. [31], who used the same Hamiltonian. This clearly shows that REM can describe the $3 \alpha+n$ system more accurately. It is interesting to note that REM gives the larger radius of the ground state despite the deeper binding energy. This means that REM yields more stretched and long-ranged wave function. It is also noted that good convergence of the $5 / 2{ }^{+}$ state was also achieved by using the same ensembles.

The left half of Fig. 3 compares the full spectrum obtained by REM and the negative-parity states calculated by Furutachi et al. [31]. Because two calculations use the same Hamiltonian, deeper binding energy means a better description of the bound states below the neutron threshold. Obviously, the present calculation gives deeper energies to all the negativeparity states below the threshold $\left(1 / 2_{1}{ }^{-}, 3 / 2_{1}{ }^{-}\right.$, and $\left.5 / 2_{1}{ }^{-}\right)$. It also gives deeper binding energy to the $7 / 2_{1}{ }^{-}$state located just above the threshold, to which the bound-state approximation may be validated. Thus, REM offers a better description of the bound states than ordinary GCM calculations.

However, the situation is different for the negative-parity resonances above the neutron threshold to which variational 


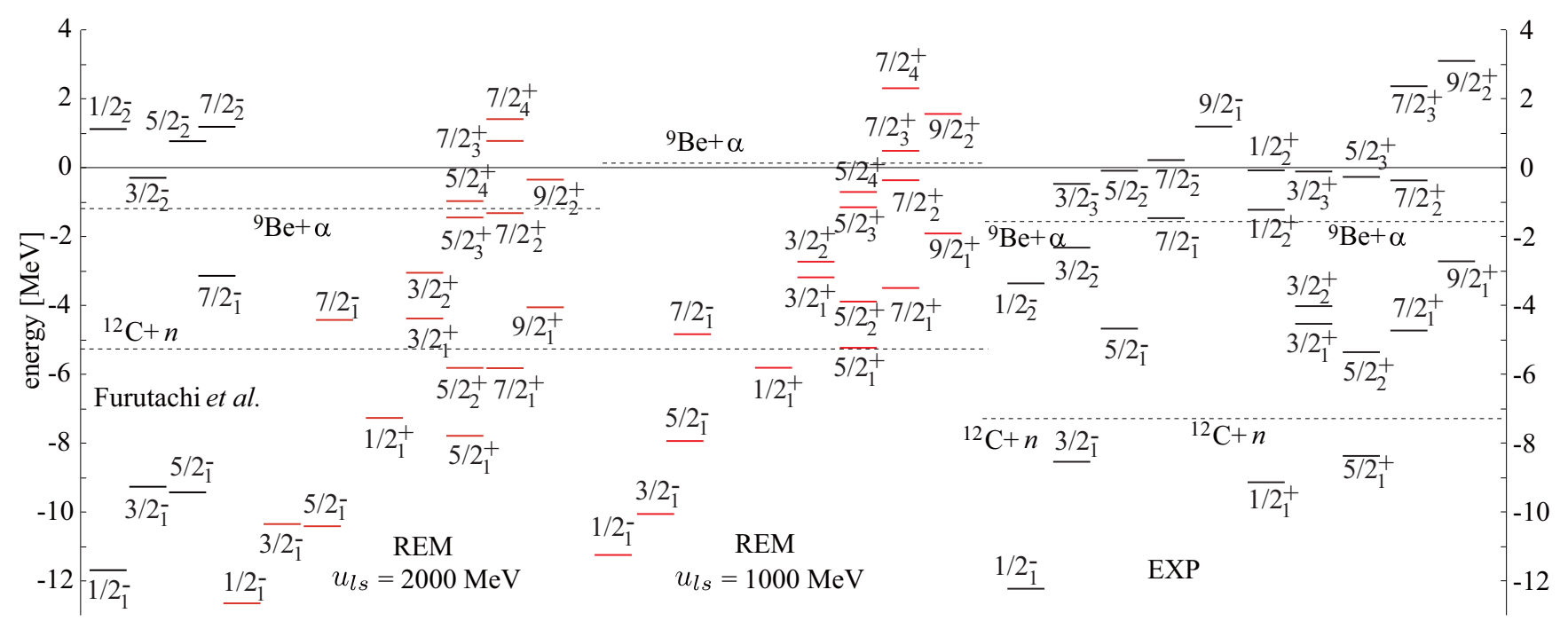

FIG. 3. The energy spectrum of ${ }^{13} \mathrm{C}$ calculated by using the strength of the spin-orbit potential $u_{l s}=2000$ and $1000 \mathrm{MeV}$. The energy is measured relative to the $3 \alpha+n$ threshold. The spectrum is compared with that obtained by Furutachi et al. [31] using the same Hamiltonian with $u_{l s}=2000 \mathrm{MeV}$. Experimental data are taken from Ref. [49].

principle is not applicable and the bound-state approximation does not guarantee the energy convergence. In fact, two calculations disagree in the highly excited negative-parity states. It is noted that the model space of REM is much larger than that of the GCM by Furutachi et al. [31]. As a result, we found that most of the negative-parity resonances are coupled with the nonresonant continuum which makes it difficult for us to identify resonant solutions from many other nonresonant solutions. Therefore, we have not shown the negative-parity states above the neutron threshold in Fig. 3. In contrast, although we cannot tell the reason clearly, we found that the coupling is not strong in the positive-parity states, and stable solutions are obtained which are plotted as resonances in the figure.

The spectrum obtained by the spin-orbit strength $u_{l s}=$ $2000 \mathrm{MeV}$ does not reproduce the order of the ground band spectrum. It underestimates the excitation energy of the $5 / 2{ }^{-}$ state and the spectrum deviates from the observed rotational pattern. This may affect the assignment of the rotational bands and the discussion of the intrinsic shape. Therefore, we performed an additional calculation using weaker spin-orbit strength $u_{l s}=1000 \mathrm{MeV}$ to check the interaction dependence of the spectrum. As seen in Fig. 3, the weaker spin-orbit strength yields the correct order of the ground band member states $\left(1 / 2_{1}{ }^{-}, 3 / 2_{1}{ }^{-}, 5 / 2_{1}^{-}\right.$, and $7 / 2_{1}^{-}$states $)$, although it still overestimates the moment of inertia of the ground band. The side effect of the weaker spin-orbit interaction is the overestimation of the excitation energies of the positive-parity states. This may be due to the overestimation of the ${ }^{9} \mathrm{Be}+\alpha$ threshold energy. If we measure them relative to the ${ }^{9} \mathrm{Be}+\alpha$ threshold, the excitation energies of many positive-parity states get closer to the observed values. This implies that many positive-parity states have ${ }^{9} \mathrm{Be}+\alpha$ structure $[29,30]$.

\section{Band assignment and shape of intrinsic states}

Figure 4 presents the band assignment determined from the calculated E2 transition strengths listed in Table I and compares it with those from the experiment and the ACM calculation. The band assignment of the REM results is unambiguous as the intraband $E 2$ transitions are clearly stronger than the interband transitions.

The $K^{\pi}=1 / 2^{-}$band is built on the $1 / 2_{1}{ }^{-}$ground state. The intraband $E 2$ transition strengths are reasonably described and comparable with the experimental data for the $1 / 2_{1}{ }^{-} \rightarrow 3 / 2_{1}{ }^{-}$and $1 / 2_{1}{ }^{-} \rightarrow 5 / 2_{1}{ }^{-}$transitions. Experimentally, the ground band terminates at the $9 / 2_{1}{ }^{-}$state, but we could not identify the corresponding state in our calculation. This may be due to the high excitation energy of this state, which causes the strong coupling with the continuum and makes it difficult to separate this state within the bound-state approximation.

For the positive-parity states, we have assigned four rotational bands: $K^{\pi}=5 / 2^{+}, 7 / 2^{+}, 1 / 2^{+}$, and $3 / 2^{+}$which are built on the $5 / 2{ }_{1}{ }^{+}, 7 / 22^{+}, 1 / 2_{1}{ }^{+}$, and $3 / 22^{+}$states, respectively. Experimentally, the $E 2$ transition strength for the $1 / 2_{1}{ }^{+} \rightarrow 5 / 2_{1}{ }^{+}$transition has already been measured (9.0 $e^{2} \mathrm{fm}^{4}$ ) [49] and our calculation gives comparable value (6.7 $\left.e^{2} \mathrm{fm}^{4}\right)$. However, no other $B(E 2)$ data are available, and the positive-parity band assignment has not been firmly established by the experiments.

In Ref. [38], based on ACM which assumes the $3 \alpha+n$ cluster structure with triangular symmetry, the authors proposed a band assignment (Fig. 4, right panel). They proposed the $K^{\pi}=1 / 2^{-}, 5 / 2^{+}$, and $7 / 2^{+}$bands which share the same intrinsic structure and the $K^{\pi}=1 / 2^{+}$and $1 / 2^{-}$bands with different structures. They also tentatively classified the observed states into the rotational bands as shown in the middle panel of Fig. 4. Their assignment is similar to the present REM results in several aspects, but there are some differences as discussed below. First, both models suggest the ground $K^{\pi}=$ $1 / 2^{-}$band and the excited $K^{\pi}=5 / 2^{+}$and $7 / 2^{+}$bands, but the REM gives much larger moment of inertia of these bands while the observed moment of inertia looks to be in between the REM and ACM results. Second, both models also suggest 


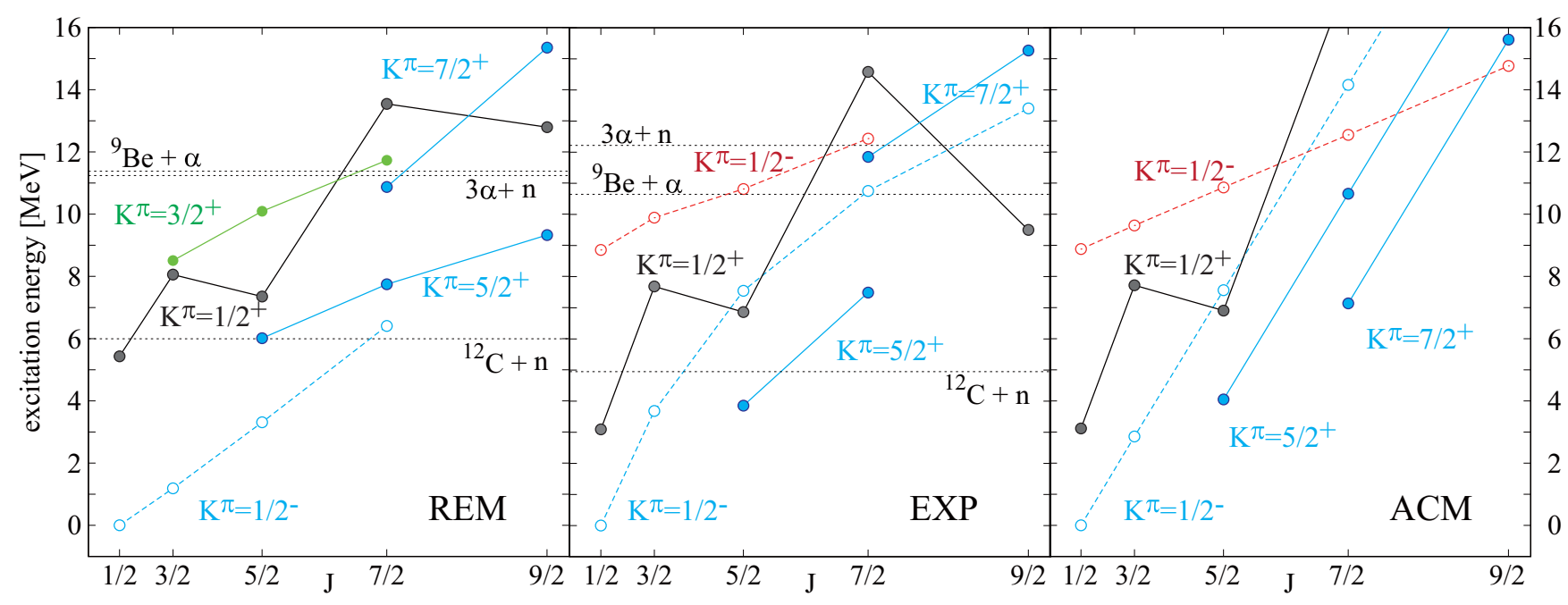

FIG. 4. The band assignment based on the calculated $E 2$ transition strengths compared with that from the algebraic cluster model (ACM) [38] and the experimental assignment, which was also tentatively proposed in Ref. [38]. The filled (open) symbols show the positive-parity (negative-parity) states.

the $K^{\pi}=1 / 2^{+}$band, but we again see the disagreement in the moment of inertia. We will examine the structure of these four bands in the following. Finally, the ACM proposed the excited

TABLE I. The calculated intra- and interband $E 2$ transition probabilities in the unit of $e^{2} \mathrm{fm}^{4}$. The transitions larger than the Weisskopf estimate $\left(1 \mathrm{~W} . \mathrm{u} .=1.8 e^{2} \mathrm{fm}^{4}\right)$ are shown. The numbers in the parentheses are the experimental values.

\begin{tabular}{|c|c|c|c|}
\hline Band $K_{i}^{\pi} \rightarrow K_{f}^{\pi}$ & $J_{i}$ & $J_{f}$ & $B\left(E 2 ; J_{i} \rightarrow J_{f}\right)$ \\
\hline \multirow[t]{5}{*}{$1 / 2^{-} \rightarrow 1 / 2^{-}$} & $1 / 2_{1}^{-}$ & $3 / 2_{1}^{-}$ & $17.4(12.7)$ \\
\hline & & $5 / 2_{1}^{-}$ & $17.1(16.9)$ \\
\hline & $3 / 2_{1}^{-}$ & $5 / 2_{1}^{-}$ & 2.4 \\
\hline & & $7 / 2_{1}^{-}$ & 17.8 \\
\hline & $5 / 2_{1}^{-}$ & $7 / 2_{1}^{-}$ & 2.0 \\
\hline \multirow[t]{3}{*}{$5 / 2^{+} \rightarrow 5 / 2^{+}$} & $5 / 2+$ & $7 / 21^{+}$ & 13.8 \\
\hline & & $9 / 21^{+}$ & 10.9 \\
\hline & $7 / 2_{1}+$ & $9 / 21^{+}$ & 12.0 \\
\hline $7 / 2^{+} \rightarrow 7 / 2^{+}$ & $7 / 2_{2}+$ & $9 / 2{ }^{+}$ & 12.9 \\
\hline \multirow[t]{5}{*}{$1 / 2^{+} \rightarrow 1 / 2^{+}$} & $1 / 2_{1}+$ & $3 / 21^{+}$ & 16.7 \\
\hline & & $5 / 2_{2}+$ & 20.1 \\
\hline & $3 / 2_{1}+$ & $5 / 2_{2}+$ & 5.0 \\
\hline & & $7 / 24^{+}$ & 7.6 \\
\hline & $5 / 2_{2}{ }^{+}$ & $9 / 2_{2}+$ & 9.9 \\
\hline \multirow[t]{3}{*}{$3 / 2^{+} \rightarrow 3 / 2^{+}$} & $3 / 2_{2}{ }^{+}$ & $5 / 2_{3}+$ & 10.0 \\
\hline & & $7 / 2{ }^{+}+$ & 8.7 \\
\hline & $5 / 2_{3}+$ & $7 / 2_{3}+$ & 9.8 \\
\hline $5 / 2^{+} \rightarrow 7 / 2^{+}$ & $7 / 2_{1}+$ & $7 / 22^{+}$ & 4.3 \\
\hline \multirow[t]{5}{*}{$1 / 2^{+} \rightarrow 5 / 2^{+}$} & $1 / 2_{1}+$ & $5 / 2_{1}^{+}$ & $6.7(9.0)$ \\
\hline & $5 / 2{ }^{+}$ & $5 / 21^{+}$ & 3.6 \\
\hline & & $7 / 21^{+}$ & 4.3 \\
\hline & & $9 / 21^{+}$ & 3.3 \\
\hline & $9 / 2_{2}{ }^{+}$ & $7 / 21^{+}$ & 2.2 \\
\hline \multirow[t]{2}{*}{$1 / 2^{+} \rightarrow 3 / 2^{+}$} & $3 / 2_{1}+$ & $7 / 23^{+}$ & 2.3 \\
\hline & $7 / 24^{+}$ & $5 / 23^{+}$ & 3.0 \\
\hline $3 / 2^{+} \rightarrow 5 / 2^{+}$ & $3 / 2_{2}+$ & $5 / 21^{+}$ & 2.7 \\
\hline
\end{tabular}

$K^{\pi}=1 / 2^{-}$band as a "Hoyle-like band," which has similar properties to the Hoyle state of ${ }^{12} \mathrm{C}$, but REM does not. This difference may be due to the bound state approximation made in the REM calculation which makes difficult to describe weakly interacting dilute resonances like the Hoyle state.

Since the REM calculation does not assume any spatial symmetry, it is interesting to investigate if there exists the triangular symmetry behind these rotational spectra. In general, the wave function of REM is a superposition of many basis wave functions with different configurations, and hence we need some measure to evaluate its intrinsic structure. For this purpose, we introduce the overlap between the REM wave function and the basis wave functions defined as

$$
O_{i}=\sum_{K K^{\prime}}\left\langle\Psi_{M}^{J \pi} \mid P_{M K}^{J \pi} \Phi_{i}\right\rangle B_{K K^{\prime}}^{-1}\left\langle P_{M K^{\prime}}^{J \pi} \Phi_{i} \mid \Psi_{M}^{J \pi}\right\rangle,
$$

where $B^{-1}$ is the inverse matrix of $B$ which is the overlap of projected basis wave functions,

$$
B_{K K^{\prime}}=\left\langle P_{M K}^{J \pi} \Phi_{i} \mid P_{M K^{\prime}}^{J \pi} \Phi_{i}\right\rangle .
$$

Note that the REM wave function $\Psi_{M}^{J \pi}$ is a superposition of $\Phi_{i}$ [Eq. (15)]. Therefore, if the overlap $O_{i}$ is large, $\Psi_{i}$ can be approximated by a single basis wave function $\Phi_{i}$.

The calculated overlaps are summarized in Table II. The ground state has the maximum overlap, which is as large as 0.83 , with the basis wave function shown in Fig. 5(a). Note that the density distribution clearly shows the triangular configuration of $3 \alpha$ particles with a valence neutron where the lengths of the triangle are 3.31, 3.30, and 3.02 fm. Furthermore, we found that all the member states of the ground band have large overlaps no less than 0.70 with the same basis wave function. Therefore, we consider that the ground band is reasonably interpreted as the rotational band having a common intrinsic structure with a triangular symmetry as asserted by Bijker et al. [38]. They also argued that the $K^{\pi}=5 / 2^{+}$and $7 / 2^{+}$bands have the same intrinsic structure and are classified as the "ground band." Indeed, we found that these bands have 
TABLE II. The calculated overlaps for each state which is defined by Eq. (16). The columns denoted by $O\left(1 / 2^{-}\right)$and $O\left(1 / 2^{+}\right)$ show the overlap between REM wave function and the basis wave function which is most dominant in the $1 / 2_{1}{ }^{-}$and $1 / 2_{1}{ }^{+}$states, respectively.

\begin{tabular}{|c|c|c|c|c|c|}
\hline \multicolumn{3}{|c|}{$K^{\pi}=1 / 2^{-}$} & \multicolumn{3}{|c|}{$K^{\pi}=1 / 2^{+}$} \\
\hline$J^{\pi}$ & $O\left(1 / 2_{1}^{-}\right)$ & $O\left(1 / 2_{1}^{+}\right)$ & $J^{\pi}$ & $O\left(1 / 2_{1}^{-}\right)$ & $O\left(1 / 2_{1}{ }^{+}\right)$ \\
\hline $1 / 2_{1}^{-}$ & 0.83 & 0.12 & $1 / 2_{1}+$ & 0.14 & 0.58 \\
\hline $3 / 2_{1}^{-}$ & 0.83 & 0.16 & $3 / 2_{1}+$ & 0.18 & 0.56 \\
\hline $5 / 2_{1}^{-}$ & 0.73 & 0.06 & $5 / 2_{2}+$ & 0.25 & 0.56 \\
\hline \multirow[t]{3}{*}{$7 / 2_{1}^{-}$} & 0.76 & 0.13 & $7 / 24^{+}$ & 0.35 & 0.25 \\
\hline & \multirow{2}{*}{\multicolumn{2}{|c|}{$K^{\pi}=5 / 2^{+}$}} & $9 / 2_{2}+$ & 0.35 & 0.45 \\
\hline & & & \multicolumn{3}{|c|}{$K^{\pi}=7 / 2^{+}$} \\
\hline$J^{\pi}$ & $O\left(1 / 2_{1}^{-}\right)$ & $O\left(1 / 21^{+}\right)$ & $J^{\pi}$ & $O\left(1 / 2_{1}^{-}\right)$ & $O\left(1 / 2_{1}{ }^{+}\right)$ \\
\hline $5 / 2_{1}+$ & 0.50 & 0.45 & $7 / 2_{2}+$ & 0.74 & 0.15 \\
\hline $7 / 2_{1}+$ & 0.54 & 0.42 & $9 / 2_{3}+$ & 0.55 & 0.19 \\
\hline \multirow{2}{*}{$9 / 2_{1}+$} & 0.58 & 0.43 & & & \\
\hline & $K^{\pi}=3 / 2$ & & & & \\
\hline$J^{\pi}$ & $O\left(1 / 2_{1}^{-}\right)$ & $O\left(1 / 2_{1}^{+}\right)$ & & & \\
\hline $3 / 2_{2}+$ & 0.45 & 0.40 & & & \\
\hline $5 / 2_{3}+$ & 0.46 & 0.26 & & & \\
\hline $7 / 2_{3}+$ & 0.35 & 0.28 & & & \\
\hline
\end{tabular}

nonsmall overlap with the same basis wave function shown in Fig. 5(a). However, our results show a deviation from a rigid shape. The magnitudes of the overlaps between these bands and the basis wave function shown in Fig. 5(a) are reduced less than 0.60 except for the $7 / 22^{+}$state. Furthermore, these bands have nonsmall overlaps with other configurations. For
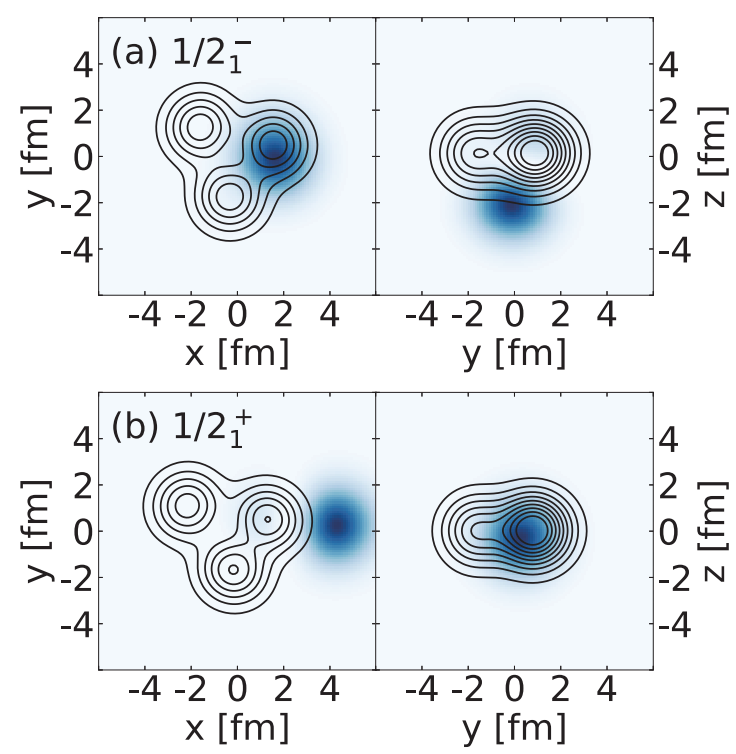

FIG. 5. (a) Density distribution of the basis wave functions which have the maximum overlap with the $1 / 2_{1}{ }^{-}$state. (b) Same as panel (a) but for the $1 / 2_{1}{ }^{+}$state. Contours show the density of $3 \alpha$ particles and color plots show the density distribution of the valence neutron wave function. example, the $K^{\pi}=5 / 2^{+}$band has large overlap with the dominant basis wave function of the $1 / 21^{+}$state, which is discussed below. Thus, the $K^{\pi}=5 / 2^{+}$and $7 / 2^{+}$bands look similar to the $K^{\pi}=1 / 2^{-}$band, but the deviation from the rigid shape is not small.

In Ref. [38], the $K^{\pi}=1 / 2^{+}$band was assigned as a rotational band which also has a triangular arrangement of $3 \alpha$ particles but has the valence neutron in a different singleparticle orbit. In the present calculation, we also found that the bandhead state $\left(1 / 2_{1}+\right.$ state $)$ has the maximum overlap with a different basis wave function whose density distribution is shown in Fig. 5(b), but has small overlap with the dominant configuration of the ground band [Fig. 5(a)]. Apparently, the position of the wave packets of the valence neutron is different from that of the $1 / 2_{1}{ }^{+}$state, and $\alpha$ particles deviate from equilateral triangular arrangement as the lengths of the triangle are $3.55,3.51$, and $2.67 \mathrm{fm}$. This confirms that the $K^{\pi}=1 / 2^{+}$ band has a different intrinsic structure. However, we again note that the magnitude of the maximum overlap is not as large as that of the ground band, and the member states of this band show the increasing mixture of other contributions as the excitation energy and angular momentum increase. In particular, the $7 / 24^{+}$state has rather small overlap with the intrinsic state of its bandhead $\left(1 / 2_{1}{ }^{+}\right.$state $)$despite the strong $E 2$ transition to another band member state $\left(3 / 2{ }_{1}{ }^{+}\right)$. This indicates that the structure of the $K^{\pi}=1 / 2^{+}$band is not as simple as a rigid rotor. Finally, we also found the strongest admixture of the various configurations in the $K^{\pi}=3 / 2^{+}$band, which is a candidate of the band proposed in Refs. [29,38]. This may be due to the highest high excitation energy of this band.

We note that the use of the stronger spin-orbit interaction $\left(u_{l s}=2000 \mathrm{MeV}\right)$ strength does not change most of the analysis discussed above. The only change caused by stronger spin-orbit interaction is the nature of the $K^{\pi}=1 / 2^{+}$band. As seen in Fig. 3, the $1 / 2_{1}{ }^{+}$state is more deeply bound by the stronger spin-orbit interaction, and hence, it tends to have more compact structure. Consequently, the overlaps $O\left(1 / 21^{+}\right)$ of the $K^{\pi}=1 / 2^{-}$and $7 / 2^{+}$bands become larger. For example, the overlap between the $1 / 21^{-}\left(7 / 2_{2}{ }^{+}\right)$state and $1 / 21^{+}$ state increases to $0.57(0.56)$. This indicates the $K^{\pi}=1 / 2^{+}$ band structure approaches rigid body due to deeper binding.

In short, the REM calculation confirmed that the ground band can be interpreted as a rigid-body rotational band which manifests the triangular symmetry. It also shows that ACM explains the general trend of the excited bands. However, we found that all the excited bands have nonsmall admixture with other configurations and deviate from the rigid-body interpretation. One of the signature of this mixing is the nonsmall E2 transitions between the bands with different intrinsic structures. Therefore, the experimental data for these transitions will provide us an important insight into the cluster structure of ${ }^{13} \mathrm{C}$.

\section{SUMMARY}

In summary, we have investigated the structure of the $3 \alpha+$ $n$ system by extending the REM framework. As a benchmark calculation for the $3 \alpha+n$ system, REM well reproduced the ground and excited energies where we followed the same 
Hamiltonian of the previous study as a comparison. It was also demonstrated that REM accurately describes the wave functions which yields to the deeper binding energies.

We have also discussed the rotational band assignment and investigated if they manifest the triangular symmetry. The proposed band assignment qualitatively explains the observed data, although the order of several bands disagrees and the $K^{\pi}=1 / 2^{-}$band is missing in the present result. From the analysis of the overlap with the basis wave functions, it was found that the ground band can be regarded as a rigid-body rotational band which manifests the triangular symmetry. We also have seen that the $D_{3 h}^{\prime}$ symmetry approximately explains the general nature of the excited bands. However, all the excited bands have nonsmall admixture with other configurations without symmetry and deviate from the rigid-body interpretation because of their high excitation energies and angular momenta. The nonsmall E2 transitions between different bands are a signature of the configuration mixing, and we expect that the experimental data for these transitions will provide us with important information about the underlying symmetry behind the observed spectrum.

\section{ACKNOWLEDGMENTS}

The authors acknowledge the fruitful discussions with Dr. Funaki and Dr. Kawabata. This work was supported by JSPS KAKENHI Grant No. 19K03859, the collaborative research programs 2020 at the Hokkaido University information initiative center, and the COREnet program at the RCNP, Osaka University.
[1] A. Tohsaki, H. Horiuchi, P. Schuck, and G. Röpke, Phys. Rev. Lett. 87, 192501 (2001).

[2] Y. Funaki, A. Tohsaki, H. Horiuchi, P. Schuck, and G. Röpke, Phys. Rev. C 67, 051306(R) (2003).

[3] T. Wakasa, E. Ihara, K. Fujita, Y. Funaki, K. Hatanaka, H. Horiuchi, M. Itoh, J. Kamiya, G. Röpke, H. Sakaguchi, N. Sakamoto, Y. Sakemi, P. Schuck, Y. Shimizu, M. Takashina, S. Terashima, A. Tohsaki, M. Uchida, H. Yoshida, and M. Yosoi, Phys. Lett. B 653, 173 (2007).

[4] M. Chernykh, H. Feldmeier, T. Neff, P. von Neumann-Cosel, and A. Richter, Phys. Rev. Lett. 98, 032501 (2007).

[5] Y. Kanada-En'yo, Prog. Theor. Phys. 117, 655 (2007).

[6] Y. Funaki, T. Yamada, H. Horiuchi, G. Röpke, P. Schuck, and A. Tohsaki, Phys. Rev. Lett. 101, 082502 (2008).

[7] M. Itoh, H. Akimune, M. Fujiwara, U. Garg, N. Hashimoto, T. Kawabata, K. Kawase, S. Kishi, T. Murakami, K. Nakanishi, Y. Nakatsugawa, B. K. Nayak, S. Okumura, H. Sakaguchi, H. Takeda, S. Terashima, M. Uchida, Y. Yasuda, M. Yosoi, and J. Zenihiro, Phys. Rev. C 84, 054308 (2011).

[8] E. Epelbaum, H. Krebs, D. Lee, and U.-G. Meißner, Phys. Rev. Lett. 106, 192501 (2011).

[9] E. Epelbaum, H. Krebs, T. A. Lähde, D. Lee, and U.-G. Meiner, Phys. Rev. Lett. 109, 252501 (2012).

[10] M. Freer, M. Itoh, T. Kawabata, H. Fujita, H. Akimune, Z. Buthelezi, J. Carter, R. W. Fearick, S. V. Förtsch, M. Fujiwara, U. Garg, N. Hashimoto, K. Kawase, S. Kishi, T. Murakami, K. Nakanishi, Y. Nakatsugawa, B. K. Nayak, R. Neveling, S. Okumura et al., Phys. Rev. C 86, 034320 (2012).

[11] Y. Fukuoka, S. Shinohara, Y. Funaki, T. Nakatsukasa, and K. Yabana, Phys. Rev. C 88, 014321 (2013).

[12] S.-I. Ohtsubo, Y. Fukushima, M. Kamimura, and E. Hiyama, Prog. Theor. Exp. Phys. 2013, 073D02 (2013).

[13] J. Carlson, S. Gandolfi, F. Pederiva, S. C. Pieper, R. Schiavilla, K. E. Schmidt, and R. B. Wiringa, Rev. Mod. Phys. 87, 1067 (2015).

[14] B. Zhou, A. Tohsaki, H. Horiuchi, and Z. Ren, Phys. Rev. C 94, 044319 (2016).

[15] N. Itagaki, S. Okabe, K. Ikeda, and I. Tanihata, Phys. Rev. C 64, 014301 (2001).

[16] J. Maruhn, N. Loebl, A. Umar, N. Itagaki, M. Kimura, H. Horiuchi, and A. Tohsaki, Mod. Phys. Lett. A 25, 1866 (2010).
[17] T. Suhara and Y. Kanada-En'yo, Phys. Rev. C 82, 044301 (2010)

[18] T. Baba, Y. Chiba, and M. Kimura, Phys. Rev. C 90, 064319 (2014).

[19] J. P. Ebran, E. Khan, T. Nikšić, and D. Vretenar, Phys. Rev. C 90, 054329 (2014).

[20] M. Freer, J. D. Malcolm, N. L. Achouri, N. I. Ashwood, D. W. Bardayan, S. M. Brown, W. N. Catford, K. A. Chipps, J. Cizewski, N. Curtis, K. L. Jones, T. Munoz-Britton, S. D. Pain, N. Soić, C. Wheldon, G. L. Wilson, and V. A. Ziman, Phys. Rev. C 90, 054324 (2014).

[21] T. Baba and M. Kimura, Phys. Rev. C 94, 044303 (2016).

[22] A. Fritsch, S. Beceiro-Novo, D. Suzuki, W. Mittig, J. J. Kolata, T. Ahn, D. Bazin, F. D. Becchetti, B. Bucher, Z. Chajecki, X. Fang, M. Febbraro, A. M. Howard, Y. Kanada-Enyo, W. G. Lynch, A. J. Mitchell, M. Ojaruega, A. M. Rogers, A. Shore, T. Suhara et al., Phys. Rev. C 93, 014321 (2016).

[23] T. Baba and M. Kimura, Phys. Rev. C 95, 064318 (2017).

[24] H. Yamaguchi, D. Kahl, S. Hayakawa, Y. Sakaguchi, K. Abe, T. Nakao, T. Suhara, N. Iwasa, A. Kim, D. H. Kim, S. M. Cha, M. S. Kwag, J. H. Lee, E. J. Lee, K. Y. Chae, Y. Wakabayashi, N. Imai, N. Kitamura, P. Lee, J. Y. Moon et al., Phys. Lett. B 766, 11 (2017).

[25] J. Li, Y. L. Ye, Z. H. Li, C. J. Lin, Q. T. Li, Y. C. Ge, J. L. Lou, Z. Y. Tian, W. Jiang, Z. H. Yang, J. Feng, P. J. Li, J. Chen, Q. Liu, H. L. Zang, B. Yang, Y. Zhang, Z. Q. Chen, Y. Liu, X. H. Sun et al., Phys. Rev. C 95, 021303(R) (2017).

[26] T. Baba and M. Kimura, Phys. Rev. C 97, 054315 (2018).

[27] P. Marević, J. P. Ebran, E. Khan, T. Nikšić, and D. Vretenar, Phys. Rev. C 99, 034317 (2019).

[28] Y. Liu, Y. L. Ye, J. L. Lou, X. F. Yang, T. Baba, M. Kimura, B. Yang, Z. H. Li, Q. T. Li, J. Y. Xu, Y. C. Ge, H. Hua, J. S. Wang, Y. Y. Yang, P. Ma, Z. Bai, Q. Hu, W. Liu, K. Ma, L. C. Tao et al., Phys. Rev. Lett. 124, 192501 (2020).

[29] M. Milin and W. Von Oertzen, Eur. Phys. J. A 14, 295 (2002)

[30] M. Freer, N. I. Ashwood, N. Curtis, A. Di Pietro, P. Figuera, M. Fisichella, L. Grassi, D. Jelavić Malenica, T. Kokalova, M. Koncul, T. Mijatović, M. Milin, L. Prepolec, V. Scuderi, N. Skukan, N. Soić, S. Szilner, V. Tokić, D. Torresi, and C. Wheldon, Phys. Rev. C 84, 034317 (2011). 
[31] N. Furutachi and M. Kimura, Phys. Rev. C 83, 021303(R) (2011).

[32] T. Suhara and Y. Kanada-En'yo, Phys. Rev. C 84, 024328 (2011).

[33] C. Wheldon, N. I. Ashwood, M. Barr, N. Curtis, M. Freer, T. Kokalova, J. D. Malcolm, V. A. Ziman, T. Faestermann, H. F. Wirth, R. Hertenberger, and R. Lutter, Phys. Rev. C 86, 044328 (2012).

[34] T. Yamada and Y. Funaki, Phys. Rev. C 92, 034326 (2015).

[35] J. P. Ebran, E. Khan, T. Nikšić, and D. Vretenar, J. Phys. G 44, 103001 (2017).

[36] Y. Chiba and M. Kimura, Phys. Rev. C 101, 024317 (2020).

[37] K. Inaba, T. Kawabata, Y. Sasamoto, M. Fujiwara, K. Hatanaka, K. Itoh, M. Itoh, K. Kawase, H. Matsubara, Y. Maeda, K. Nakanishi, K. Suda, S. Sakaguchi, Y. Shimizu, A. Tamii, Y. Tameshige, M. Uchida, T. Uesaka, and H. P. Yoshida, JPS Conf. Proc. 31, 011069 (2020).

[38] R. Bijker and F. Iachello, Phys. Rev. Lett. 122, 162501 (2019).
[39] R. Bijker and F. Iachello, Phys. Rev. C 61, 067305 (2000).

[40] V. Della Rocca, R. Bijker, and F. Iachello, Nucl. Phys. A 966, 158 (2017).

[41] R. Bijker and F. Iachello, Prog. Part. Nucl. Phys. 110, 103735 (2020)

[42] R. Imai, T. Tada, and M. Kimura, Phys. Rev. C 99, 064327 (2019).

[43] B. Zhou, M. Kimura, Q. Zhao, and S.-h. Shin, Eur. Phys. J. A 56, 298 (2020).

[44] A. B. Volkov, Nucl. Phys. 74, 33 (1965).

[45] N. Yamaguchi, T. Kasahara, S. Nagata, and Y. Akaishi, Prog. Theor. Phys. 62, 1018 (1979).

[46] D. M. Brink, Proc. Int. School of Physics Enrico Fermi, Course 36, Varenna, edited by C. Bloch (Academic Press, New York, 1966).

[47] D. L. Hill and J. A. Wheeler, Phys. Rev. 89, 1102 (1953).

[48] J. J. Griffin and J. A. Wheeler, Phys. Rev. 108, 311 (1957).

[49] F. Ajzenberg-Selove, Nucl. Phys. A 523, 1 (1991). 